\title{
Educación emprendedora: Estado del arte
}

\author{
Entrepreneurial Education: State of the Art
}

\section{José Carlos Sánchez García ${ }^{a^{*}}$, Alexander Ward, Brizeida Hernández \& Jenny Lizette Florez}

Universidad de Salamanca, Salamanca, España.

aProfesor, investigador y catedrático del emprendimiento, experto a nivel internacional en psicología social de las organizaciones y director de la Cátedra de Emprendedores, desde donde coordina programas de formación en emprendimiento. Dirige activamente, el grupo de investigación IDEM y la Red AFIDE-

EMPRENDE.

\author{
Recibido 16-07-17 \\ Aprobado 01-10-17 \\ En Línea 10-08-17
}

\section{Correspondencia}

Email: jsanchez@usal.es

\section{Citar como:}

Sanchez, J.C., Ward, A., Hernández, B., \& Florez, J. (2017). Educación emprendedora: Estado del arte. Propósitos y Representaciones, 5(2), 401 - 473. doi: http://dx.doi.org/10.20511/pyr2017.v5n2.190

(c) Universidad San Ignacio de Loyola, Vicerrectorado de Investigación, 2017.

(cc) BY-NC-ND Este artículo se distribuye bajo licencia CC BY-NC-ND 4.0 Internacional (http://creativecommons.org/licenses/by-nc-nd/4.0/). 


\section{Resumen}

La incertidumbre y ambigüedad en el empleo es una realidad en muchas sociedades actuales, por lo cual la idea de auto emplearse ha comenzado a tomar un rol más activo, no solo por necesidad, sino por los prospectos que trae de innovación y mejoría a las sociedades. Ante esta situación social nos asalta la pregunta: ¿Cómo podemos potenciar el espíritu emprendedor en nuestros estudiantes? El artículo tiene como objetivo recopilar información teórica del estado del arte sobre la educación emprendedora; lo que ha sido, es, y puede ser, con la finalidad de presentar la actualidad sobre el tema, tanto en el campo psicológico como en el campo pedagógico. Para lograr este objetivo, se recopiló información proveniente de 108 fuentes investigativas, refiriéndose a libros y artículos de revista, concluyendo que, a pesar de que a nivel general la educación emprendedora ha contribuido significativamente a su entorno y la producción de empresas, países tercermundistas o subdesarrollados, específicamente los de Latinoamérica, aun requieren esfuerzo extra para implementar en su cabalidad el tema de emprendimiento a nivel académico, particularmente, por desafíos como inestabilidad política, tecnología y competencia a nivel internacional.

Palabras clave: Educación, Emprendimiento, Latinoamérica, Psicología, Pedagogía

\section{Summary}

Job uncertainty and ambiguity is a reality in many current societies, therefore, the idea of self-employment has taken a more active role, not only out of necessity, but also because it brings innovation and development to societies. In view of this situation, the following question arises: how can we strengthen the entrepreneurial spirit of our students? This article has the objective to collect theoretical information of the state of the art on entrepreneurship education: what it has been, what it is, and what can it be, in order to present the current condition of the topic, both in the psychological and pedagogical 
field. To achieve this objective, information from 108 research sources, mainly from scientific articles and books was collected, concluding that, although generally, entrepreneurship education has significantly contributed to the creation of businesses, developing countries, specifically, those in Latin America, still require extra effort to fully implement entrepreneurship topic in the curriculums, particularly because of challenges such as political instability, technology and international competition.

Keywords: Education, Entrepreneurship, Latin America, Psychology, Pedagogy, 


\section{Introducción}

El emprendimiento siempre ha estado presente en el trascurso de la historia de la humanidad, pues es inherente a ésta. En las últimas décadas este concepto se ha vuelto de suma importancia, ante la necesidad de superar los constantes y crecientes problemas económicos. Dicho esto, la formación en competencias emprendedoras se vuelve un elemento imprescindible para la adaptabilidad de los nuevos mercados laborales. Siendo el estudio del emprendimiento un potente escenario de investigación, nace el interés de ver cómo se puede impulsar el mismo por medio de programas educativos orientados a esta meta: educación emprendedora.

El fenómeno del emprendimiento, denominado por el término anglosajón "entrepreneurship", es un área de creciente desarrollo en el campo de la investigación científica. El interés académico en torno al emprendimiento se basa en la evidencia acerca de su contribución al crecimiento económico, al rejuvenecimiento del tejido socio-productivo, al relanzamiento de los espacios regionales, a la dinamización del proceso innovador y a la generación de nuevos puestos de trabajo (Kantis, Ishida \& Komori, 2002).

El presente trabajo tiene como meta recopilar una revisión teórica y de artículos científicos que permiten evidenciar la realidad actual sobre la educación emprendedora, así como la relevancia que tiene la Psicología en este campo y las distintas estrategias ofrecidas desde esta ciencia al fortalecimiento de las habilidades y competencias requeridas, por la comunidad educativa, a la hora de decidir emprender. Además, pretende recordar la complejidad del fenómeno educativo, así como la aplicación de las distintas estrategias de aprendizaje que permitan fortalecer las competencias y habilidades de los alumnos que le permitan considerar las opciones de emprender. Para lograr este objetivo, se recopiló información proveniente de 107 fuentes investigativas, refiriéndose a libros y artículos de revista. Para evaluar el estado actual de la educación en los países latinoamericanos, se utilizaron fuentes que tuvieran menos de 20 años, de las cuales los programas 
investigados fueron verificados para corroborar su función y vigencia. Las bases de datos utilizadas para consultar la literatura fueron ProQuest, Scopus, además de reportes o libros realizados por agencias gubernamentales de ministerios de educación, la Comisión Europea y GEM.

Los artículos fueron propiamente organizados según las temáticas que se describen en este artículo: Teorías psicológicas sobre emprendimiento, Perfil estudiantil y sus actitudes emprendedoras, Estrategias de educación enfocadas en emprendimiento, Rol actual de las instituciones educativas en emprendimiento, Futura dirección de la educación emprendedora, Actualidad de educación emprendedora en Latinoamérica. Las conclusiones se realizaron evaluando los artículos más recientes, y se contrastaron con la literatura científica que predice éxito en montar y manejar empresas, con la meta de crear un panorama sobre la actualidad de educación emprendedora según su correspondencia a la literatura científica y a las realidades que representa hoy día.

\section{Argumentación.}

\section{Trasfondo teórico.}

A partir de las diferentes revisiones realizadas en base a estudios bibliométricos (entre otros, Busenitz et al., 2003; Cornelius, Landström, \& Persson, 2006; Dery \& Toulouse, 1994; Ferreira, Nuno \& Rui, 2015; Filion, 1997; Landström, Harirchi \& Åström, 2012; Murphy, Liao, \& Welsch, 2006) se puede formar un panorama de las diferentes teorías que se han venido desarrollando en torno al emprendimiento en tres grandes enfoques o perspectivas teóricas (Sánchez, 2011a): la económica, la psicológica y la sociológica. A nuestro entender, el campo del emprendimiento se situaría en la intersección de estas tres perspectivas. 


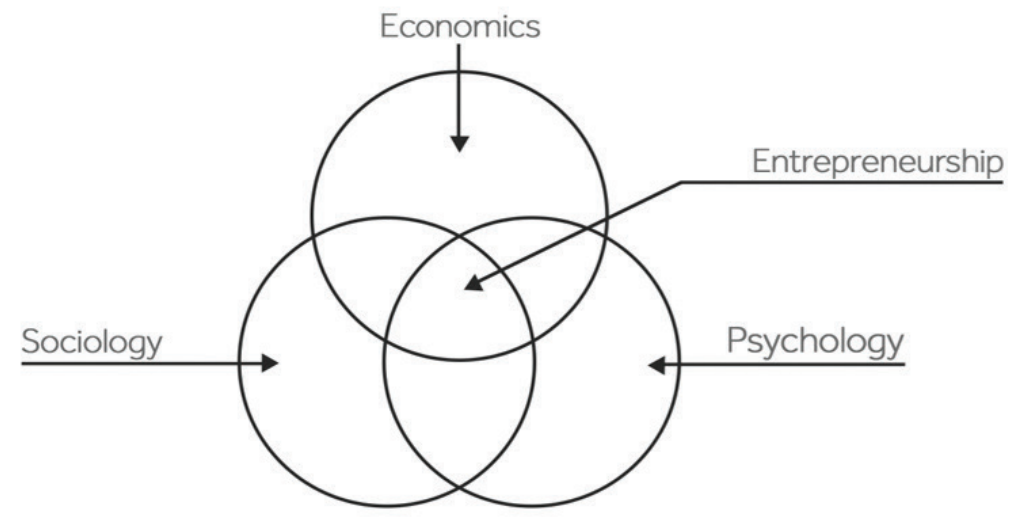

Figura 1. Marco conceptual del emprendimiento como campo de conocimiento. Fuente: Sánchez 2011, p.429

Para poder comprender la relevancia de la Educación Emprendedora y el aporte de la Psicología, es crucial resaltar la importancia del emprendimiento en la sociedad. Uriarte y Martín (2007), respecto al desarrollo de nuevas empresas, consideran elemental para el crecimiento económico del país y las instituciones impulsar a los jóvenes hacia el autoempleo y la creación su propia empresa. En este sentido, la Psicología, tal como lo afirman estos autores, debería profundizar en el estudio de los comportamientos económicos de los empresarios, así como en los motivos, cogniciones, actitudes, valores y todas aquellas características psicológicas que subyacen para que, en una población determinada y dadas unas condiciones objetivas fijas, se decida a trabajar por cuenta propia, asumiendo altos riesgos económicos a la hora de ponerse al frente de una organización.

A esto se agrega lo que plantea Sánchez (2010), quién considera que en las últimas décadas ha habido un aumento considerable, tanto en el volumen como en la sofisticación, de estudios que exploran cuestiones relacionadas con el emprendimiento, con la creación de empresas. En gran parte, esto se da a razón que las empresas de tamaño pequeño y mediano han mostrado ser importantes para la economía, además de ser los principales agentes del empleo y del crecimiento económico de algunos países. Mucho de este 
esfuerzo por entender el fenómeno del emprendimiento se ha centrado en las características psicológicas de las personas que emprenden estas aventuras.

Es aquí donde la consolidación de las estrategias de aprendizaje y el aporte de la Psicología a este campo adquieren relevancia, siendo los enfoques psicológicos sobre el emprendimiento los que han experimentado, recientemente, una revitalización por la importancia futura de los emprendedores a pequeña escala, y porque su estudio está en la frontera entre la psicología del trabajo (personalidad, actividades laborales, etc.), psicología organizacional (fundadores de la organización que tienen una enorme influencia sobre ella), y la psicología de mercados (actividades económicas en el mercado). Esencialmente, todos los aspectos de la psicología están implicados al estudiar el campo del emprendimiento (Rauch \& Frese, 2007).

Teniendo en cuenta lo anterior, es importante resaltar el perfil emprendedor y los avances de este mismo perfil con base en las competencias requeridas para que los alumnos del ámbito universitario puedan llegar a autoemplearse, las cuales tienden a ser fortalecidas a través de los distintos programas de las universidades donde se potencian dichas competencias; es importante, señalar el aporte de la Psicología a todo este proceso de potenciación necesarios para incentivar el comportamiento emprendedor en los jóvenes universitarios.

Un aspecto importante que se debe tener en mente cuando se habla de la educación emprendedora es que está integrado de forma sistemática como misión en el campo educativo. Las instituciones académicas están creadas para el servicio de la sociedad, y se ha fundamentado tres misiones para este objetivo: educación, por medio de la docencia, investigación, y la tercera y de nuestro mayor interés: aporte al desarrollo económico, por medio de la tecnología de empresas o la creación de mismas por parte del alumnado y docencia.

Cuando se habla de la tercera misión, se habla de varios fundamentos conceptuales, donde al menos se pueden identificar tres: primero desarrollo 
de actividades de cooperación entre universidad y agentes públicos y privados; segundo, compromiso social y comunitario a la solución de problemas de su alrededor (Bueno \& Casani, 2007), y tercero el desarrollo de universidades emprendedoras (Clark,1998) y (Etzkowitz,2004). Este papel de la universidad debe estar directamente vinculado a las realidades específicas de cada sociedad; los enfoques de la misma coinciden en que la misma tenga apertura hacia su realidad social, contraste la idea una imagen de torre de marfil, por encima o aislada de los problemas sociales (Vera, Amaru \& González, 2013).

\section{Enfoque en perfil emprendedor desde teorías psicológicas.}

A medida que la psicología ha tomado auge dentro de la comunidad científica, particularmente, en función de su integración interdisciplinaria, ha contribuido su grupo de teorías prevalecientes al momento de identificar actitudes emprendedoras: Teoría de los Rasgos de Personalidad, Teoría Cognitiva, Teoría de la Acción Razonada, Teoría del Comportamiento Planificado, entre otras toman el centro del pilar del campo. En síntesis:

Teoría de los Rasgos de Personalidad: Parte del supuesto que los emprendedores poseen valores en la personalidad que difieren de los noemprendedores. A pesar de ser un clásico en emprendimiento, ha tenido debates sobre su utilidad a través del tiempo. Cromie (2000) y Hisrich (2000), por ejemplo, han encontrado que la personalidad no diferencia de manera fiable entre los que emprenden y no emprenden, además de mostrar un porcentaje pequeño en varianza para éxito. No obstante, un estudio por Sánchez y Yurrebaso (2005) encontró que las características personales si funcionan como variables predictoras de intención emprendedora, además de explicar gran parte de su varianza. En particular, autoeficacia y locus de control interno impactan significativamente en la intención emprendedora de estudiantes (Sesen, 2013). 
Teoría Cognitiva: Supone que los emprendedores poseen una estructura de conocimiento que utilizan para hacer evaluaciones, juicios, o decisiones que implica la evaluación de oportunidades, la creación de negocios y el crecimiento de las mismas" (Mitchell et al., 2002; Sánchez, 2011a).

Teoría de la Acción Razonada: Considera que la acción en las personas es, en su gran parte, fundamentada en estados racionales, utilizando la información disponible de forma sistémica para hacer un juicio valorativo sobre sus implicaciones. En esencia, conceptualiza la intención como un precursor a la acción, aunque no pretende establecer que intención siempre lleva acción; no tienen una correspondencia perfecta (Gallurt Plá, 2010). El grado de intención depende, según la teoría, de la actitud (como la persona valora la acción), y la norma subjetiva (como la percibe según la valoración de otros significantes).

Teoría del Comportamiento Planificado: Propuesta por Ajzen (1991), revisa la Teoría de Acción Razonada, manteniendo la actitud y norma subjetiva como elementos imprescindibles para la acción emprendedora, pero agregando control percibido, formando una teoría más completa, cual corrige las limitaciones de la previa sobre comportamiento y falta de control.

Dentro de esta esfera, se va creando un perfil formado por varios factores influénciales, tales como personalidad, apoyo social, sistema económico, cultura y valores. Muchos investigadores han encontrado que rasgos de personalidad como auto-eficacia, locus de control interno y proactividad en niveles altos influencian significativamente a tomar acción emprendedora, además de mostrar compromiso a sus actividades (Gurel, Altinay, \& Daniele 2010; Iakovleva, Kolvereid \& Stephan 2011; Koh 1996; Mauer, Neergard, \& Kirketerp 2009; Prabhu \& Poulose 2012). No obstante, por personalidad solamente no se puede explicar la acción de emprender, sino que existe un factor cognitivo envuelto en la actitud emprendedora. En efecto, estudios han afirmado que creencias y valores individuales influencian comportamiento emprendedor (Krueger \& Brazeal 1994; Krueger \& Carsrud 1993). Son este conjunto de rasgos, valores y cogniciones los que probablemente 
llevan a las personas a autoemplearse (Learned 1992), junto el trasfondo sociocultural, cual funciona como estimulante o motivador (Mueller \& Thomas 2001). En otras palabras, un emprendedor podría ser una interacción de factores personales: Autoeficacia, locus de control interno y proactividad; y su trasfondo sociocultural: actitud, control percibido, norma subjetiva y fiabilidad.

\section{Perfil estudiantil y actitudes emprendedoras.}

Actitudes, de acuerdo con la teoría de comportamiento planificada, son precursores a intención, cuales son antecedentes al comportamiento (Ajzen, 1991). Este componente de comportamiento se trata de predisposiciones e intenciones para actuar de una forma particular hacia un evento (Shaver, 1987). Shapero y Sokol (1982) sugieren que de estas actitudes se deriva intención emprendedora. De aquí, ¿qué actitudes se pueden asociar a emprendimiento y educación, de forma que permita crear un perfil emprendedor en estudiantes?

En la actualidad, los valores comportamentales en la variable estudiantil emprendedora son inconclusos, no obstante, se utiliza generalmente los mismos que se dan en el ámbito general de empresarios: necesidad de logro, locus de control, disponibilidad a tomar riesgos, tolerancia a la ambigüedad, innovación y autoconfianza. Es decir, al momento de establecer un perfil emprendedor en esta población, se utiliza como supuesto que poseen estas características. Estudios específicos han profundizado y mostrado de forma empírica la prevalencia de algunas de estas actitudes en el ambiente académico. Hatten y Ruhland (1995), por ejemplo, encontraron que estudiantes de empresa cuales poseen locus de control interno desarrollaron actitudes positivas hacia emprendimiento luego de participar en programas de iniciativa de empresas pequeñas en varias universidades.

Al momento de determinar la dinámica demográfica en la actitud emprendedora, se ha encontrado que los hombres son dos veces más propensos que las mujeres a iniciar actividades empresariales, y en parte podría ser 
por el desarrollo de actitudes en experiencias previas. La prevalencia investigativa ha indicado que mujeres (Hisrich \& Brush, 1987; Carter, 2000; Thomas, 2001) y minorías (Kourilsky \& Esfandiari, 1997; Heilman \& Chen, 2003) enfrentan obstáculos en desarrollo emprendedor, tales como menos experiencia, recursos limitados y menos mentores. Tomando como fundamento la idea que el proceso emprendedor es experiencial por naturaleza (Robinson, Stimpson, Huefner \& Hunt, 1991; Sullivan, 2000; Minniti \& Bygrave, 2003; Politis, 2005), se podría entender por qué hay una diferencia marginal entre estos grupos demográficos y actitud emprendedora.

Aplicado al ámbito educativo, la necesidad de establecer programas que propicien eventos y ambientes que fomenten actitudes emprendedoras podrían actuar como agente de cambio para contrarrestar este problema, ya que la actitud emprendedora son una serie de valores que se nutren de situaciones propensas a fomentarla (Robinson, Stimpson, Huefner, \& Hunt, 1991; Mitra \& Matlay, 2004), y que su retro comunicación positiva aumenta las expectativas emprendedoras (Gatewood, Shaver, Power \& Gartner, 2002).

Suplementario a esto, se pueden identificar contextos que fomentan actitudes emprendedoras en los estudiantes. Muchos adultos jóvenes, particularmente, entre las edades 25 y 34, están interesados en la creación de negocios y son más propensos de entablar actividades emprendedoras (Minniti, Allen \& Langowitz., 2005), son edades sensitivas donde se puede capitalizar en la actitud, utilizando el rango de intereses como inercia para acelerar la integración de dichos valores al individuo.

\section{¿Cómo se puede fomentar la educación emprendedora en profesores y estudiantes?}

Uno de los mayores retos que tiene el sistema educativo es establecer los mecanismos adecuados para que la innovación y el emprendimiento sean considerados fundamentales en el proceso educativo en todos los niveles de enseñanza. Además de promover la formación del profesorado en 
metodologías que permitan el desarrollo de la innovación en los procesos de enseñanza y en el aprendizaje.

El desafío particular que tiene la educación en emprendimiento es poder convertir las ideas en acción. Métodos tradicionales, tales como lecturas, revisión de literatura, exámenes, entre otros, no activan el emprendimiento (Gibb, 2002; Sogunro, 2004). Un estudio encontró que incluso inhiben el desarrollo de las actitudes y competencias emprendedoras (Kirby, 2002). Por tal, al momento de adecuar otras técnicas para mediar el aprendizaje en base a competencias, hay que tomar no solo una coyuntura que abarque conocimiento científico, sino que también promueva la creatividad en no solo pensamiento, también cultura del esfuerzo, emprendimiento, toma de decisiones, trabajo en equipo, análisis y solución de problemas, comunicación, creatividad, innovación a lo largo de su permanencia y promoción del sistema educativo, con la finalidad de contar con ciudadanos emprendedores que generen un impacto social y económico sobre el futuro del país.

Para ello se requiere del uso de estrategias efectivas que permitan evidenciar de manera clara el aprendizaje significativo centradas en el aprendizaje experiencial y vivencial, que se enfocan en la construcción del conocimiento en contextos reales, en el desarrollo de las capacidades reflexivas, críticas y en el pensamiento de alto nivel, así como en la participación en las prácticas sociales auténticas de la comunidad de acuerdo a Díaz (2003) y que se pueden relacionar con el fortalecimiento de las competencias propias de una persona emprendedora, las políticas educativas deben enfocarse en estimular las aptitudes emprendedoras a través de nuevas formas de enseñar y aprender desde la educación primaria, además de prestar una atención particular, en la enseñanza secundaria. Ello exige el esfuerzo de las direcciones escolares y la formación del profesorado para asegurarse de que los estudiantes tengan en las escuelas oportunidades de vivir experiencias de emprendimiento prácticas.

Los profesores y maestros juegan un papel primordial: son facilitadores del aprendizaje y multiplicadores de ideas, y ayudan al estudiante a conseguir 
resultados en el aprendizaje relacionados con el emprendimiento como conocimiento, capacidades y aptitudes. Como mínimo, todo profesor ha de acceder durante su carrera a una experiencia de formación en los temas y métodos claves relativos al aprendizaje emprendedor y la educación en emprendimiento. Las competencias emprendedoras requieren métodos activos para involucrar a los estudiantes en la creatividad y la innovación.

Otro factor de suma importancia es que las competencias y las aptitudes emprendedoras solo se pueden adquirir o construir mediante experiencias prácticas de aprendizaje de la vida real. Estas experiencias se pueden integrar en todas las asignaturas del programa curricular. Los profesores y la escuela no podrán cumplir estas metas a menos que trabajen en cooperación y asociación y con estrecha relación con la comunidad.

Para conseguir todo lo anterior, es necesario: Una buena formación inicial del profesorado, para que inspiren a sus estudiantes desde el principio de su carrera profesional y programas de formación con estrategias y visión de emprendimiento. La educación en emprendimiento se puede integrar como un enfoque horizontal a lo largo de todo el programa de estudio usando pedagogías contemporáneas como, por ejemplo:

Aprendizaje centrado en la solución de problemas auténticos: Consiste en la presentación de situaciones reales o simulaciones auténticas vinculadas a la aplicación o ejercicio de un ámbito de conocimiento o ejercicio profesional (dado el caso de la educación superior), en las cuales el alumno debe analizar la situación y elegir o construir una o varias alternativas viables de solución. Para algunos autores incluye el aprendizaje mediante el análisis y resolución de casos, las estrategias de simulación y juegos, además de tener un enfoque altamente investigativo.

Metodología basada en proyectos: Propone elaborar proyectos como enfoque escolar general; planes que se llevan a cabo desde la planificación de centro, con el fin de lograr un servicio o producto único, mediante una serie de tareas y uso efectivo de recursos. El enfoque de proyectos 
puede abarcar al currículo y a la enseñanza de manera conjunta, pero lo importante es que esté organizado alrededor de actividades desde una perspectiva experiencial, donde el alumno aprende a través de la experiencia personal, activa y directa con el fin de iluminar, reforzar y asimilar el aprendizaje cognitivo.

Trabajo en equipos cooperativos: Se basa en la utilización didáctica de grupos pequeños, con finalidad de optimizar aprendizaje y el de los otros integrantes (Johnson, Johnson, \& Smith, 1991a). De acuerdo a Lillo, (2013) es importante recalcar que el aprendizaje que busca generar este método no se da necesariamente de forma natural al dar una instrucción o tarea a un grupo de alumnos, ya que debe existir a la base una intencionalidad que dé paso, a través del trabajo en equipo, al aprendizaje deseado o planificado. Para que haya cooperación hay que asegurar cinco elementos fundamentales (Yániz \& Villardón, 2006): interdependencia positiva, responsabilidad individual y social, interacción, habilidades sociales y de pequeño grupo, y autoevaluación del proceso grupal.

El desarrollo de programas de gran calidad para el desarrollo profesional es esencial, para esto es necesario una escuela en la que se valore el espíritu emprendedor y que cuente con el respaldo de un equipo directivo eficaz. Redes de enseñanza emprendedora para garantizar la continuidad de la calidad y compartir experiencias, por ejemplo, co-aprendizaje entre docentes y alumnos, donde el alumno se vuelve autor de su aprendizaje y el maestro funciona como mediador o catalítico son de utilidad (Fiet, 2000b). No obstante, la efectividad de estos va entrelazado a cuan integrado está a estos pilares de la educación. En principio, cualquiera es capaz de emprender si es dado la oportunidad de ser asertivo, creativo e innovador; todas asequibles en un salón de clases (Fiet, 2000b). Para lograr este objetivo, es necesario crear un entorno que permita la innovación en la formación y la práctica docente, conjunto métodos de evaluación avanzados y de calidad garantizada en la pedagogía de la educación en el emprendimiento y, como punto más 
importante: debatir, reflexionar y evaluar sus programas de aprendizaje en lo referente a métodos y conocimientos ¿cómo se aprende lo que se aprende?

\section{¿Qué rol juegan las instituciones educacionales en emprendimiento?}

La educación emprendedora es, presentemente, una realidad en constante progreso. Esta contribuye a formación de una cultura (empezando por los más jóvenes y niños); impulsando estas actitudes y capacidades emprendedoras, se beneficia a la sociedad, incluso más allá de la aplicación de éstas a nuevas iniciativas empresariales, esto en Unión Europea y con mucha fuerza inicia en América Latina.

Efectivamente, la educación emprendedora ha tomado prioridad en las universidades (Matlay, 2008; Busenitz et al., 2003; Hannon, 2006; Heinonen \& Poikkijoki, 2006; Klein and Bullock, 2006; Kuratko, 2005), y se ha encontrado según algunos estudios una relación significativa entre programas emprendedores e intención emprendedora en sus alumnos (Gupta, Turban, Wasti, \& Sikdar, 2009; Guerrero, Rialp, \& Urbano, 2008; De Pillis \& Reardon, 2007; Fayolle, Gailly, \& Lassa-Clerc, 2006; Sanchez, 2013; Zhao, Seibert, \& Hills, 2005).

Un programa académico de emprendimiento podría definirse como cualquier programa o proceso educativo que se utiliza para desarrollar actitudes, destrezas y competencias emprendedoras, con el fin de desarrollar las cualidades requeridas para crear nuevos negocios (Fayolle et al., 2006). Generalmente, la educación emprendedora que se ve hoy se categoriza en tres distintos tipos:

Educar sobre emprendimiento: Impartir conocimiento general sobre conceptos relacionados a emprendimiento (Kuip \& Verheul, 2003; O’Connor, 2013).

Educar para emprender: Acercamiento teorético y práctico para proveer conocimiento y habilidades emprendedoras (Heinonen \& Hytti, 2010; Scott, Rosa, \& Klandt, 1998). 
Educar a través de emprendimiento (Action-based): Método fundamentado en el proceso y experimental, donde potenciales emprendedores aprenden directamente por medio de experiencias emprendedoras (Kyrö, 2005).

Además, a estas, Liñán (2004) formuló diferentes tipos de programas de educación emprendedora:

Educación para concientizar emprendimiento: Programa dedicado a Impartir conocimiento sobre emprendimiento e influenciar las actitudes hacia intenciones emprendedoras.

Educación para Start-Up: Programa para encarrilar personas cuales ya tienen ideas de negocio hacia resolver problemas prácticos e impulsarlos a la fase Start-Up.

Educación para dinamismo emprendedor: Programa orientado a promover personas que ya son emprendedoras y desean incorporar planes dinámicos luego de la fase Start-Up.

Educación continua para emprendedores: Programas de aprendizajes continuos para emprendedores que han exitosamente montado negocios y tienen algún tipo de expertis.

Siguiendo la misma línea, las instituciones académicas han creado programas o cursos fundamentándose en estas categorías, principalmente, en la educación práctica (Action-based), cual ha tenido el foco más alto en literatura emprendedora, por su naturaleza experiencial (Henry, Hill \& Leitch, 2005; Lackéus, Lundqvist, Williams, 2013; Rasmussen \& Sörheim, 2006), y ha consecuentemente seguido expandiéndose en su definición (Lackéus et al., 2013; Lackéus \& Williams Middleton, 2015; Lackéus, 2013).

A continuación, se dan algunos ejemplos sobre buenas prácticas en programas de emprendimiento:

Finlandia, Universidad de Jyvaskyla: curso introductorio Ciudadanía participativa y emprendimiento para profesores de primaria. 
Bélgica, Escuela de educación de Lovaina: proyecto e iniciativas para mejorar las prácticas docentes creativas y emprendedoras.

Reino Unido, Escuela Universitaria St. Mary: licenciatura en formación del profesorado que promueve la educación en emprendimiento.

Estados Unidos: programa de aprendizaje emprendedor, formación en economía, perfil basado en el trabajo.

España, Fundación Princesa de Girona: curso Formación del Talento Emprendedor en el que participan más de 300 escuelas.

España Ashoka: escuelas Changemaker, proyecto de innovación educativa para entender el ecosistema educativo (formación docente, espacios de aprendizaje, metodología, cultura de centro, participación de/en la comunidad, evaluación).

Un objetivo más integrado a la idea de educar sobre emprendimiento es el desarrollo de Universidades Emprendedoras. Centrado a la idea emprendedora, éstas intentan funcionar como focos de educación, cuales promuevan estas actitudes además de funcionar como incubadoras de empresas, proveyendo a los alumnos con nuevas ideas, competencias y habilidad de pensar de forma emprendedora hacia las demandas sociales. Tradicionalmente, las instituciones se enfocan en el campo investigativo $\mathrm{y}$ en educar; las universidades emprendedoras visualizan un tercer enfoque: la comercialización de nuevos conocimientos para desarrollos económicos (Etzkowitz, Webster, Gebhardt, Brance \& Cantisano 2000). En este sentido, la universidad toma un rol crucial en educar propiamente a personas que potencian ser emprendedores, tomando la vanguardia en desarrollar capacidades de tomar decisiones intuitivamente, autonomía, networking, toma de iniciativas, identificación de oportunidades, creatividad, pensamiento estratégico, autoeficacia, manejar situaciones impredecibles y las actitudes y formas de pensar, sentir, comunicar organizar y aprender de los emprendedores (The Entrepreneurial University: From concept to action, 2013). 
Por tal, uno de los retos del sistema educativo es propiciar precondiciones relacionadas al autoempleo. Esta reorientación del proceso educativo puede llevar a la utilización de las instituciones educativas como incubadoras de empresas., una herramienta útil para comenzar nuevos negocios, spinoffs y construir nexos con las industrias (EC/OECD, 2012), implicando a los alumnos en entrenamientos. A continuación, la Tabla 1 provee algunas características que debe tener una universidad para ser considerada emprendedora:

\section{Tabla 1.}

Caracteristicas de las universidades emprendedoras, según la Comisión Europea (2012), Thorsp \& Goldstein (2010) y Maribel Guerrero et al. (2015).

$\begin{array}{ccc}\text { Comisión Europea y foro } & \text { Holden Thorsp y Buck } & \text { Maribel Guerrero et al. } \\ \text { OECD LEED } & \text { Goldstein }\end{array}$

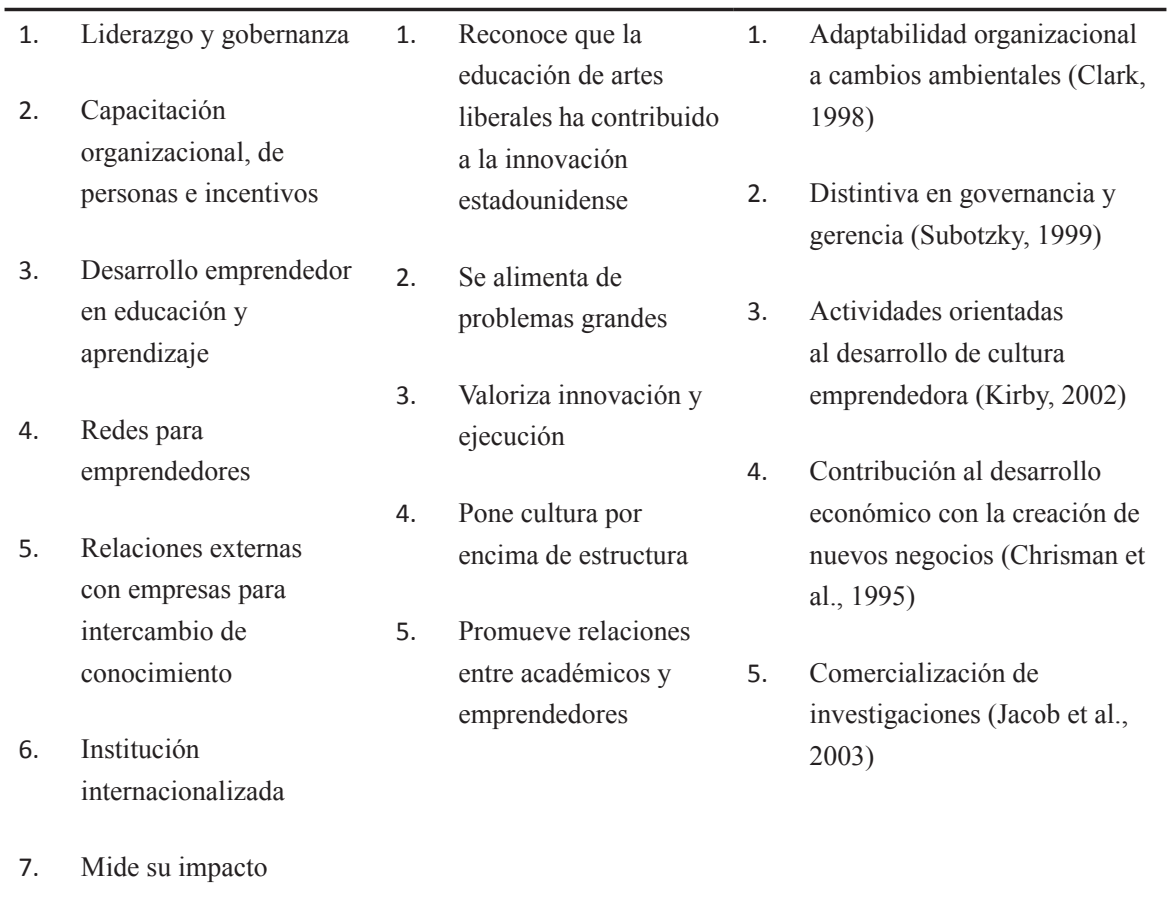

Fuente: Bikse, Lusena-Ezera, Rivza, Volkova (2016) 
En síntesis, la universidad debe integrar el emprendimiento en cada aspecto de ella; demostrar excelencia en liderazgo en todos sus niveles, facultades innovadoras con impacto tangible en sus recursos que imparten la educación, una fuerte implicación con sus alumnos junto a la diversidad en oportunidades de aprendizaje, de negocios y comunidad local, además de mostrar un compromiso a largo plazo de desarrollar el emprendimiento y formar empresas (Arnaut, 2010). De acuerdo con este mismo autor, esta transformación de universidades tradicionales a emprendedoras seria integrar el desarrollo social y económico en toda su dimensión, asegurando un estándar de educación superior.

\section{¿A dónde nos dirige la educación emprendedora?}

La implementación de temas emprendedores en el currículo académico ha sido un proceso, es decir, algo que se ha ido construyendo progresivamente durante el transcurso del tiempo. A pesar de tener su grado de éxito, es un plan que sigue creciendo $\mathrm{y}$, un elemento importante que debe tener la educación emprendedora es su adaptabilidad a las demandas sociales, en otras palabras, la técnica para realizarlo debe ser tan transformacional para el negocio como para la pedagogía (Bikse, et al., 2016).

Proponentes como Besong y Holland (2015), sugieren que la Educación para la Sustentabilidad es una buena forma de asegurar el desarrollo de destrezas, conocimientos y valores que promuevan un modo de operar cual, humanísticamente, lleve a la mejoría de calidad de vida sin destruir el medioambiente para futuras generaciones. De igual forma, David y Bell (2016) proponen que los alumnos aprendan a dirigir sus destrezas e innovaciones hacia la economía verde, con metas de empresas sostenibles.

Rico, Cámara, y Llamazares (2015), investigaron el impacto de la educación emprendedora y encontraron que ambos, emprendedores y educadores, reclaman que debe haber formación sobre cómo gestionar empresas, específicamente con conocimientos sobre marketing, contabilidad, o economía. Por otro lado, los expertos del tema creen en la necesidad 
de formación en conocimientos transversales como planes de empresa, negociación y desarrollo de proyectos. Cuando lo aplicamos a la educación formal, los currículos no deben limitarse solo a potenciar actitudes en los alumnos, sino que también formarlos con el conocimiento técnico de saber efectivamente manejar una empresa. Las Universidades que han implementado programas de educación emprendedora han evidenciado progresos. Algunos ejemplos son la Universidad de Stanford, o el Instituto de MIT en Cambridge, donde han podido integrar de forma interdisciplinaria el emprendimiento, o han utilizado su especialidad académica para crear programas que ayuden a impulsar alumnos en esa dirección; Stanford, con su programa Mayfield Fellows, ha impulsado sobre 52 alumnos a formar empresas, de las cuales diez han sido adquiridas por empresas grandes como Google hasta el 2001 (Eesley \& Miller, 2012). La Universidad de Twente ha creado sobre 200 empresas (Hopkin, 2001), y el Instituto de Tecnología de Massachusetts ha creado sobre 4,000 empresas con ganancias de \$232 billones, generando sobre 1.1 millones de empleos. Estos ejemplos son el rumbo que deberá estar tomando la educación emprendedora, a la medida que exitosamente se vaya integrando en la academia.

Otra vía para el futuro de la educación emprendedora es la creación de programas temáticos; que se enfoquen en sectores, industrias o en alguna tecnología particular, en vez de simplemente dar una idea general sobre emprender. El Instituto de Ciencias Sociales de Tata en Mumbai es un ejemplo de este cambio cual podría concretar la educación emprendedora, donde su Máster en Emprendimiento Social trata específicamente sobre competencias tales como mantener intacto el valor del depositario y no sólo el valor del accionista (Joshi, 2014). Este mismo investigador sostiene que está ocurriendo un cambio de paradigma, donde la investigación de emprendimiento está cambiando no solo a pensar como entrenar, educar y desarrollar, sino como deben ser educados para generar más impacto.

Un punto esencial en este proceso es que no todos los países realizan los cambios al mismo ritmo. Algunos ya han puesto en marcha una estrategia 
global de educación en emprendimiento en todos los niveles educativos, mientras que aún otros se encuentran en la línea de salida. Por consiguiente, las iniciativas de implementación serán diferentes a cada situación.

Además, se puede ir viendo un efecto en la creación de empresas de modo formal (registradas legalmente y reguladas) versus informal (ilegales y sin registrar). Un estudio realizado por Jiménez, Palmero-Cámara, GonzálezSantos, González-Bernal, y Jiménez-Eguizábal (2015) encontró que la educación emprendedora terciaria incrementa emprendimiento formal, a razón de niveles altos de autoconfianza, bajo riesgo percibido y aumento de capital, mientras que disminuye el informal por estar concientizado de las repercusiones negativas, cual podría mostrar un aumento en el futuro hacia las formales a la medida que se continúe integrando emprendimiento en los currículos.

\section{Latinoamérica: ¿Cuál es su actualidad en educación emprendedora?}

Compuesto de un diverso panorama de regímenes políticos y realidades sociales, Latinoamérica ha tomado sus iniciativas en fortalecer el campo de emprendimiento en currículos académicos cada vez de forma más evidente en algunos países más que otros. Por la misma lógica, cada cual ha intentado en función a sus problemas y necesidades específicos en reforzar el emprendimiento por medio de programas que se ajustan a sus situaciones y valores culturales como, por ejemplo, un panorama altamente enfatizado en educación en Argentina, a pesar de ser lo opuesto en República Dominicana o México, cuales no han logrado impregnar el valor emprendedor de la misma forma (Herrera \& Yong, 2004). Por otro lado, existen países como Perú, donde el espíritu de emprender es alto, a pesar de carecer los fundamentos básicos que determinan el emprendimiento exitoso, como un trasfondo educacional fuerte que desarrolle las competencias (Villarán de la Puente, 2014). Esta sección se dedicará principalmente en acentuar las modalidades educativas en emprendimiento que toman la vanguardia en 
distintos países de Latinoamérica, enfocándose en esos que se imparten en escuelas y universidades.

Según el análisis realizado por Herrera y Yong (2004), países como Costa Rica, El Salvador, Guatemala, Honduras, Nicaragua, Panamá, México y la República Dominicana tienen pocos esfuerzos educativos en contraste a otros países como Colombia o Brasil, observando carencias en el diseño curricular sobre elementos imprescindibles en el comportamiento emprendedor, tales como responsabilidad, autonomía, trabajo en equipo, adaptabilidad, manejo de recursos, redes sociales, toma de riesgos y aprender a emprender. No obstante, ha habido acciones fuertes para contrarrestar esta realidad. Por ejemplo, México cuenta con programas que permiten a los alumnos consolidar sus proyectos con objetivos de negocio por medio del eje curricular Toca (2010). Algunos de estos han obtenido la certificación Incubadora de Empresas Tradicionales, lo cual permite dar respaldo a microempresarios, servicios de información y asesoría en planes de negocio, registro de empresas, orientaciones para obtención de financiamiento, entre otros (De Xena, 2012). La secretaria de economía, a través del Instituto Nacional del Emprendedor (INADEM), ha creado la Universidad del Emprendedor, donde emprendedores y empresarios pueden acceder a cursos y herramientas en línea, que les servirán para fortalecer sus conocimientos, habilidades y capacidades con meta de materializar sus ideas, hacer crecer o consolidar los negocios. A nivel primario y secundario, México tiene muy pocas propuestas para la inclusión didáctica del mismo en sus currículos (Damian, 2015), aunque ha estado recientemente en proceso de mejoría. Un programa creado para afrentar esta situación, Mi Primera Empresa: emprender jugando, por la Asociación Nacional de Universidades e Instituciones de Educación Superior (ANUIES) y la Fundación Educación Superior-Empresa (FESE), intenta proporcionar conocimientos básicos a los niños por medio de un proyecto de creación de una empresa infantil, del cual se espera que aprendan sobre el comportamiento y cultura emprendedora. El mismo ha mostrado ser efectivo en demostrar que los niños son capaces 
de adquirir y aplicar conocimiento teorico sobre emprendimiento (Damian, 2015). Fuera del país, el Estado Mexicano ha formado aceleradoras de empresas (Aceleradoras Tecnológicas de Negocios), con fin de promover la internacionalización comercial del país (Kantiss, 2011).

Países como Argentina, Bolivia, Chile, Paraguay y Uruguay son países los cuales, al evaluar las fuentes que promueven el espíritu emprendedor por sectores: civil, público y privado, se ha encontrado anteriormente que el sector más implicado en desarrollar el espíritu emprendedor en los jóvenes es el de la sociedad civil, con 75 programas implementados $(71,4 \%$ de todos los programas en los tres sectores). Le sigue el sector público, con 19 $(18,10 \%) \mathrm{y}$, finalmente, está el sector privado con 11 programas $(10,48 \%)$ (Fernández, 2004). Estos resultados son relevantes porque, dentro de la sociedad civil, las universidades son las que mayormente están promoviendo el desarrollo emprendedor en los jóvenes de estos países, junto a incubadoras de empresas, también la mayoría proviniendo de las universidades. De igual forma, hay que considerar factores como la tasa de alumnos que asisten a universidades, y como viable alternativa en estos países, se propone la de incorporar formalmente la enseñanza en escuelas públicas, que podrían abarcar a jóvenes que no pueden acceder a la educación universitaria (Fernández, 2004).

Brasil fue en algún punto uno de los países más emprendedores del mundo, según el Servicio Brasileño de Apoyo a las Micro y Pequeñas Empresas (Sebrae), donde casi el 13\% de la población adulta era emprendedora para el 2003, desde entonces ahora ha pasado un tiempo considerable que ha hecho que Brasil ya no sea considerado como tal. Muchos de los programas para impulsar el emprendimiento están orientados hacia la juventud, por medio de programas de capacitación e incentivos para empresas. En el ámbito de la educación emprendedora, el Ministerio de Educación tiene dos programas prominentes con fines a estas metas: Programa Técnico Emprendedor, orientado hacia escuelas técnicas/profesionales, y Programa Educación Emprendedora para la Educación Media. El primero tiene 
como objetivo diseminar cultura emprendedora a fines de crear iniciativa en ambos profesores y alumnos, que desarrollen iniciativa, solución de problemas y trabajar buscando resultados. Este programa tiene sobre 35 incubadoras asociadas al mismo desde el 2004. El segundo tiene como meta la integración del emprendimiento en las aulas educacionales de todas las disciplinas. De igual forma, el programa Projovem está orientado a estimular las competencias emprendedoras, por medio de apoyo que permita llevarlos a crear autoempleo; este se fundamenta en los proyectos estudiantiles, y no en los currículos.

Colombia, Ecuador, Perú y Venezuela comenzaron a mostrar signos de educación emprendedora a finales de la década de los noventa (Lozano, 2004). Colombia cuenta con centros dentro de sus universidades para desarrollar cultura empresarial de carácter innovador y desde la perspectiva de responsabilidad social, trabajando áreas relacionadas al espíritu empresarial, tales como cultura, creación de empresas, formación de líderes empresariales, educación emprendedora, gestión de empresas familiares y gestión de pymes (De Xena, 2012). Además de programas para fortalecer el ambiente empresarial en las áreas específicas del país.

Venezuela, según Pietrosemolli (2006), la sociedad ha mostrado atraso en emprendimiento por no haber creado una ventana que permita no solo que surjan, sino que se fomente el espíritu empresarial fuerte, creativo y creciente; un precio que pagaran futuras generaciones. De Xena (2012) llama a un rol más participativo de parte del Estado, individuos y empresas para que se planifique, integren y coordinen hacia la obtención de objetivos de desarrollo nacional coherentes y sostenibles para contrarrestar la situación sociopolítica del mismo. De la misma forma, su estudio concluye que el sector educativo es consciente de la necesidad, apostando al desarrollo del país por gerentes en educación empresarial, a pesar de no ser un objetivo que el Estado directamente apoya.

Perú es un país con una tasa alta de actividad emprendedora, en efecto, múltiples veces considerado uno de los países más emprendedores del 
mundo, incluyendo en reportes del GEM. En el reporte del 2012, obtuvo una actividad emprendedora total (TEA) de 23\%, ubicándose en el tercer lugar, luego de China y Chile que tienen una TEA de $24 \%$, cada uno. No obstante, esto se traduce a nivel práctico en gran parte a microempresas informales que no son capaces de sostenerse. El rol que ha jugado la educación emprendedora en el país es más silente, en contraste a otros países latinoamericanos, para contrarrestar la creación de miles de microempresas de baja productividad. Esto no significa que no hay actividad educativa, varios programas han intentado contrarrestar ese estatus.

Villarán de la Puente (2014) comenta que mucho fundamento utilizado para eficazmente implementar el emprendimiento en la educación depende de cuan bien se pueden manejar problemas en educación superior y universidades en el país, cual tiene un régimen legal de autonomía extrema, dificultando la aplicación de políticas públicas, además de falencias en educación técnica. Por tal, proponen que el emprendimiento se imparta desde la educación básica.

En cuanto a la educación emprendedora, Perú cuenta con varias universidades cuales imparten desarrollo de competencias emprendedoras, generalmente con la meta de expandirse de forma internacional en el fomento del emprendimiento y la innovación, además de fomentar el espíritu empresarial en alumnos de cursos de empresariado. De igual forma, ha logrado esfuerzos cuales permite a los alumnos explorar ambientes empresariales ambos dentro y fuera del país, cuales ofrezcan la oportunidad de entrevistar con empresarios líderes de la zona, conocer gremios y cámaras de comercio de la región (Lozano, 2004). De esta forma, mantienen una impresión latente de modelos emprendedores cuales les permite llevar y adaptar al país de vuelta.

La Educación Básica Regular (EBR) tiene como meta, según el Diseño Curricular Nacional de Educación Básica Regular (2008), de "desarrollar actividades laborales y económicas que le permitan organizar su proyecto de vida y contribuir al desarrollo del país", además de "desarrollar aprendizajes 
en las ciencias, humanidades, la técnica, así como aquellos que permitan al educando un buen uso y usufructo de las nuevas tecnologías". Es decir, por esto, que se abre una ventana para la educación emprendedora. En particular, Villarán de la Puente (2014) encontró dos elementos dentro de sus objetivos cuales directamente son orientados al mismo: el desarrollo de la capacidad productiva, innovadora y emprendedora como parte de la construcción del proyecto de vida de todo ciudadano, y el desarrollo de la creatividad, innovación, apreciación y expresión a través de las artes, las humanidades y las ciencias.

De la misma forma, el Ministerio de Educación del Perú (2009) indica que la educación secundaria trabaja las siguientes actitudes en sus alumnos: disposición emprendedora. disposición y confianza en sí mismo. voluntad y automotivación para el logro de sus metas. autonomía para tomar decisiones y actuar. disposición para trabajar cooperativamente y disposición para liderar. valora la biodiversidad del país y se identifica con el desarrollo sostenible, todas cuales forman parte del núcleo del comportamiento emprendedor.

En luz de ver estos prospectos educativos sobre emprendimiento, una manera de ver la situación positivamente, comenta Villarán de la Puente (2014), es aprovechar la alta energía de emprender ya existente en el país como impulso para implementar la educación emprendedora, de forma más cabal, y acaparar el interés existente de autoempleo con tal de crear microempresas viables con alta productividad, formales y puedan sostenerse económicamente.

En Sánchez y Hernández (2016) encontramos un estudio sobre los indicadores de la Educación Emprendedora en Latinoamérica, en concreto los países de Argentina, Ecuador, México, Panamá, Perú y Uruguay. De destacar las conclusiones a las que llegan estos autores que demuestran claramente que el nivel de educación emprendedora de estos países es un buen predictor de su tasa de actividad emprendedora, manifestando así la importancia que la educación emprendedora tiene para el desarrollo emprendedor de los países implicados. 


\section{Conclusiones}

La educación emprendedora, indiscutiblemente, ha progresado desde que comenzó a conceptualizarse en escuelas de negocio como cursos a mediados del siglo pasado. Desde eso, a su integración en la Universidad de South Carolina como una concentración, a su expansión en el mapa global durante los 1980's ha dado un rumbo completo en su estado de desarrollo, donde de un curso optativo, se convirtió en programas, a actualmente existir en universidades como un elemento completamente integrado por medio de currículos transversales.

No hay duda de que el emprendimiento es una potente herramienta para afrontar las dificultades de empleo que enfrenta la juventud en múltiples sociedades a nivel global, además de ser una fuente de creatividad e innovación que contribuye significativamente al desarrollo de sociedades. No obstante, luego de revisar la literatura y explorar el trasfondo en países latinoamericanos, es esencial poder crear un sistema que oriente y apoye el desarrollo de la mentalidad y espíritu emprendedor. El emprendimiento no debe encararse como simplemente la solución para el desempleo entre los jóvenes, ya que tiende a presentar elevadas tasas de fracaso, sino que debe utilizarse como una forma de concientizar lo que significa emprender y lo que es necesario para serlo efectivamente (Furtado, 2003). El mismo debe estar hecho para cambiar el "chip" mental de los jóvenes, con el fin de que no solo piensen en el autoempleo como un proyecto de trabajo, sino como una forma de mejorar su desarrollo y el de la sociedad. Las variables psicológicas asociadas a éxito, como factores personales: Autoeficacia, locus de control interno y proactividad; y su trasfondo sociocultural: actitud, control percibido, norma subjetiva y fiabilidad deben ser siempre puestas como fundamento en las propuestas que se realizan para promover la cultura emprendedora a nivel educativo, más aun cuando se ha mostrado que influencian en la creación de empresas formales. 
Como respuesta a la pregunta inicial: ¿Cómo podemos potenciar el espíritu emprendedor en nuestros estudiantes? aprender a emprender, el quinto pilar de la educación, toma un rol esencial para impulsar la intención emprendedora a materializar en realidades exitosas y sostenibles. Particularmente, Latinoamérica, que, a pesar de ser una región de países con recursos abundantes, la realidad actual le trae desafíos constantes tales como tecnología y competencia a nivel internacional. Con la llegada de ambos, es una oportunidad del currículo emprendedor adaptarse a esta modalidad, permitiendo crear sistemas o programas que le den la oportunidad al alumno de interactuar con el exterior, con tal de aprender y diversificar sus técnicas y pensamientos empresariales, adaptándolos a las realidades de su país. Debe cada país reconocer y detectar quienes tienen potencial de emprender y desarrollarlos con espíritu de tomar acción, ya que la solución de problemas económicos depende de la propia fuerza laboral del país. En resumen, identificar oportunidades de desarrollo emprendedor y capitalizar en ellas.

De la misma forma, no se puede medir de la misma manera la eficacia operacional entre países desarrollados y subdesarrollados. Muchos lugares a nivel global no cuentan con acceso a educación tan fácil como en países desarrollados, por tal, los programas de educación en emprendimiento no deberían estar orientados solo hacia universidades privadas o públicas, sino a cualquier institución capaz de impartir educación, tales como las escuelas públicas o privadas, desde primaria a secundaria. La educación emprendedora se vuelve un proyecto de todos los sectores sociopolíticos de un país, y donde se enfocan los programas debe ser bajo el mismo principio: donde sea que se pueda educar. Considerando los valores emprendedores pueden desarbolarse desde temprana edad, es fuertemente recomendado que comiencen desde que el niño comienza a educarse.

El peso de la labor de crear programas de educación emprendedora no está solo en desarrollarlos, sino en mantenerlos. Estos deberían constantemente ser evaluados para medir su efectividad y viabilidad en la creación de competencias y el valor de intención emprendedora en alumnos. No solo 
esto, sino que, para realmente ser un pilar en la educación, deben estar integrados en toda su cabalidad dentro de los diversos currículos académicos. La educación emprendedora debe trascender su condición optativa, y ser compulsiva dentro de la educación, de modo que se cree un formato de educación transversal, de otro modo, solo se atenderá superficialmente el emprendimiento.

Finalmente, algo que se debe tener en consideración es que la predisposición de emprender esta directamente atado al rol de la cultura y viceversa, convirtiéndose en el agente que crea y promueve el espíritu emprendedor. El que emprende debe ser recodificado a impactar directamente el desarrollo de su realidad; que sus valores emprendedores estén relacionados a una motivación intrínseca, pero a la vez atada a la cultura que experimenta, así sea capaz de crear un cambio también a nivel extrínseco. Solo así la educación emprendedora habrá realmente tenido éxito.

\section{Referencias}

Ajzen, I. (1991). The theory of planned behavior. Organizational Behavior \& Decision Processes, 50(2) 179-211. https://doi.org/10.1016/07495978(91)90020-T

Anzola R, S. (2004). El nuevo rostro empresarial: indagación sobre el empresariado juvenil en América Latina y el Caribe. Banco Interamericano de Desarrollo. Bogotá: Alfaomega.

Arnaut, D. (2010). Towards an Entrepreneurial University. International Journal of Euro-Mediterranean studies, 3(1). Recuperado de: http:// www.emuni.si/press/ISSN/1855-3362/3_135-152.pdf

Besong, F. \& Holland, C. (2015). The dispositions, abilities and behaviours (DAB) Framework for profiling learnersí sustainability competencies in higher education. Journal of Teacher Education for Sustainability, 17(1), 5-22. https://doi.org/10.1515/jtes-2015-0001 
Bikse, V., Lusena-Ezera, I., Rivza, B., Volkova, T. (2016). The Transformation of Traditional Universities into Entrepreneurial Universities to Ensure Sustainable Higher Education. Journal of Teacher Education for Sustainability, 18(2), 75-88. https://doi.org/10.1515/jtes-2016-0016

Bueno, E. \& Casani, F. (2007). La tercera misión de la Universidad: enfoques e indicadores básicos para su evaluación. Economía Industrial, 366, 4359.

Busenitz, L.W., West III, G.P., Shepherd, D., Nelson, T., Chandler, G.N., $\&$ Zacharakis, A. (2003). Entrepreneurship research in emergence: Past trends and future directions. Journal of Management, 29(3), 285-308. https://doi.org/10.1016/S0149-2063(03)00013-8

Carter, S. (2000). Improving the numbers and performance of womenowned businesses: some implications for training and advisory services. Education + Training, 42(4/5), 326-34. https://doi. org/10.1108/00400910010373732

Clark, B. (1998). Creating Entrepreneurial Universities: Organizational Pathways of Transformation. International Association of Universities and Elsevier Science Ltd., New York and Oxford.

Cornelius, B., Landström, H., \& Persson, O. (2006). Entrepreneurial studies: The dynamic research front of a developing social science. Entrepreneurship Theory and Practice, 30(3), 375-398. https://doi. org/10.1111/j.1540-6520.2006.00125.x

Cromie, S. (2000). Assesing entrepreneurial inclinations: some approaches and empirical evidence. European Journal of Work and Organizational Psychology, 9(1), 7-30. https://doi.org/10.1080/135943200398030

David V., \& J. Bell. (2016). Twenty first century education: Transformative education for sustainability and responsible citizenship. Journal of Teacher Education for Sustainability, 18(1), 48- 56.

De Pillis, E., \& Reardon, K. (2007). The influence of personality traits and persuasive messages on entrepreneurial intention: a cross-cultural comparison. Career Development International, 12(4), 382-96. https:// doi.org/10.1108/13620430710756762 
De Xena, L. B. (2012). La educación empresarial en instituciones de educación superior venezolanas. Estudios Gerenciales, 28(125), 51-58. https://doi.org/10.1016/S0123-5923(12)70007-4

Dery, R., \& Toulouse, J. M. (1994). La restructuration sociale du champ de l'entrepreneurship: le cas du Journal of Business Venturing. Cahier de recherche- Ecole des hautes études commerciales. Chaire d'entrepreneurship Maclean Hunter.

Díaz, F. (2003). Cognición situada y estrategias para el aprendizaje significativo. Revista Electrónica de Investigación Educativa, 5(2). Recuperado de: https://redie.uabc.mx/redie/article/view/85

Ministerio de Educación. (2008). Diseño Curricular Nacional de Educación Básica Regular. Ministerio de Educación, Lima.

EC/OECD. (2012). A Guiding Framework for Entrepreneurial Universities. Recuperado de: https://www.oecd.org/site/cfecpr/EC-OECD\%20 Entrepreneurial\%20Universities\%20Framework.pdf

Eesley, C., \& Miller, W, (2012). Impact: Stanford University's Economic Impact via Innovation and Entrepreneurship, Stanford University. Recuperado de: https://engineering.stanford.edu/sites/default/files/ Stanford_Alumni_Innovation_Survey_Report_102412_1.pdf

Etzkowitz, H. (2004). The evolution of the entrepreneurial university. International Journal of Technology and Globalization, 1(1), 64-77. https://doi.org/10.1504/IJTG.2004.004551

Etzkowitz H., Webster A., Gebhardt C., Brance R., \& Cantisano T. (2000). The future of the University and the University of the future: Evolution of ivory tower to entrepreneurial paradigm. Research Policy, 29(2), 313330. https://doi.org/10.1016/S0048-7333(99)00069-4

European Commission. (2012). Entrepreneurship in the EU and beyond. Flash Euro-barometer Report 354.

Fayolle, A., Gailly, B., \& Lassa-Clerc, N. (2006). Assessing the impact of entrepreneurship education programmes: a new methodology. Journal of European Industrial Training, 30(9), 701-720. https://doi. org/10.1108/03090590610715022 
Fernández C., M. A. (2004). El nuevo rostro empresarial: indagación sobre el empresariado juvenil en América Latina y el Caribe. Banco Interamericano de Desarrollo. Bogotá: Alfaomega.

Ferreira, M., Nuno R. \& Miranda, R. (2015). Thirty years of entrepreneurship research published in top journals: analysis of citations, co-citations and themes. Journal of Global Entrepreneurship Research, 5 (17), 1-22. https://doi.org/10.1186/s40497-015-0035-6

Fiet, J.O. (2000b). The pedagogical side of entrepreneurship theory. Journal of Business Venturing, 16 (2), 101-17.https://doi.org/10.1016/S08839026(99)00042-7

Filion, L.J. (1997). From Entrepreneurship to Entreprenology. In S. Kunkel (Ed.), Entrepreneurship: The Engine of Global Economic Development. Journal of Best Papers of the 42nd World Conference, International Council for Small Business. San Francisco.

Furtado, A. (2003). Empreendedorismo - Jovens - Experiências Internacional e Brasileira - Impactos Sobre o Emprego. Consultoria LegislativaEstudo.

Gallurt Plá, P. (2010). Creación de «spin-offs» en las universidades españolas: un modelo de intenciones (Tesis doctoral). Universidad Pablo de Olavide, España.

Gatewood, E.J., Shaver, K.G., Powers, J.B., \& Gartner, W.B. (2002). Entrepreneurial expectancy, task effort, and performance. Entrepreneurship Theory \& Practice, 27(2), 187-206. https://doi. org/10.1111/1540-8520.00006

Gibb, A.A. (2002). In pursuit of a new 'enterprise' and 'entrepreneurship' paradigm for learning: creative destruction, new values, new ways of doing things and new combinations of knowledge. International Journal of Management Review, 4(3), 233-69. https://doi.org/10.1111/14682370.00086

Global Entrepreneurship Monitor Consortium. (2012). GEM Global Entrepreneurship Monitor: Global Reports. Recuperado de: http://www. gemconsortium.org/report.

Guerrero M., Cunningham JA., \& Urbano D. (2015). Economic impact of entrepreneurial universities activities: An exploratory study of the United 
Kingdom. Research Policy, 44, 748- 764. https://doi.org/10.1016/j. respol.2014.10.008

Guerrero, M., Rialp, J., \& Urbano, D. (2008). The impact of desirability and feasibility on entrepreneurial intentions: a structural equation model. International Entrepreneurship and Management Journal, 4(1), 35-50. https://doi.org/10.1007/s11365-006-0032-x

Gupta, V.K., Turban, D.B., Wasti, S.A., \& Sikdar, A. (2009). The role of gender stereotypes in perceptions of entrepreneurs and intentions to become an entrepreneur. Entrepreneurship Theory Practice, 33, 397417.https://doi.org/10.1111/j.1540-6520.2009.00296.x

Gurel, E., Altinay, L., \& Daniele, R. (2010). Tourism students' entrepreneurial intentions. Annals of Tourism Research, 37(3), 646-669.https://doi. org/10.1016/j.annals.2009.12.003

Hannon, P., (2006), Teaching Pigeons to Dance: Sense and Meaning in Entrepreneurship Education, Education + Training, Special Issue on Entrepreneurship Education, 48(5), 296-308.

Hatten, T.S., \& Ruhland, S.K. (1995). Student attitude towards entrepreneurship as affected by participation in an SBI program. Journal of Education for Business, 70(4), 224-228. https://doi.org/10.1080/0883 2323.1995.10117754

Heinonen, J., \& Poikkijoki, S. (2006). An Entrepreneurial-directed Approach to Entrepreneurship Education: Mission Impossible? Journal of Management Development, 25(1), 80-94. https://doi. org/10.1108/02621710610637981

Heinonen, J., \& Hytti, U. (2010). Back to basics: the role of teaching in developing the entrepreneurial university. The International Journal of Entrepreneurship and Innovation, 11(4), 283-292. https://doi. org/10.5367/ijei.2010.0006

Henry, C., Hill, F. M., \& Leitch, C. M. (2005). Entrepreneurship education and training: can entrepreneurship be taught? Part I. Education + Training, 47(2), 98 - 111.https://doi.org/10.1108/00400910510586524 
Herrera Amighetti, C., \& Yong Chacón, M. (2004). El nuevo rostro empresarial: indagación sobre el empresariado juvenil en América Latina y el Caribe. Banco Interamericano de Desarrollo. Bogotá: Alfaomega.

Heilman, M.E., \& Chen, J.J. (2003). Entrepreneurship as a solution: the allure of self-employment for women and minorities. Human Resource Management Review, 13(2), 347-364. https://doi.org/10.1016/S10534822(03)00021-4

Hisrich, R.D. (2000). Marketing. (2a ed.). Hauppauge, NY: Barrons.

Hisrich, R.D., \& Brush, C.G. (1987). Women entrepreneurs: a longitudinal study. in Ronstadt, R., Hornaday, J.A., Peterson, R. and Vesper, K.H. (Eds), Frontiers of Entrepreneurship, Babson College, Wellesley, MA, pp. 187-9.

Hopkin, D. (2001). The Role of Universities in the Modern Economy. Institute of Welsh Affairs, Denbigh, National Eisteddfod Lecture.

Iakovleva, T., Kolvereid, L., \& Stephan, U. (2011). Entrepreneurial intentions in developing and developed countries. Education + Training, 53(5), 353-370. https://doi.org/10.1108/00400911111147686

Jiménez, A., Palmero-Cámara, C., González-Santos, M. J., Gonzalez-Bernal, J., \& Jiménez-Eguizábal, J. A. (2015). The impact of educational levels on formal and informal entrepreneurship. BRQ Business Research Quarterly, 18(3), 204-212. https://doi.org/10.1016/j.brq.2015.02.002

Junqueira Botelho, A. J., Jonathan, E. \& Gallagher, T. (2004). El nuevo rostro empresarial: indagación sobre el empresariado juvenil en América Latina y el Caribe. Banco Interamericano de Desarrollo. Bogotá: Alfaomega.

Johnson, David W., Johnson, Roger T., \& Smith, Karl A. (1991a). Cooperative learning: Increasing college faculty instructional productivity. ASHEERIC Report on Higher Education. Washington, DC: The George Washington University.

Joshi, R. (2014). Entrepreneurship education: Core, context and challenges. Journal of Entrepreneurship and Management, 3(2). 27-36. 
Kantiss, H. (2011). Políticas y programas de desarrollo emprendedor: El estado del conocimiento en América Latina. Desarrollo, innovarión y cultura empresarial, 3, 82-100.

Kantis, H., Ishida, M., \& Komori, M. (2002). Empresarialidad en economías emergentes. Creación de empresas en América Latina y el Este de Asia. Banco Interamericano de Desarrollo y Universidad Nacional de General Sarmineto: Buenos Aires,

Kirby, D. (2002). Entrepreneurship education: can business schools meet the challenge? Paper presented at the RENT XVI Conference, Barcelona, 21-22 November.

Kyrö, P. (2005). Entrepreneurial learning in a cross-cultural context challenges previous learning paradigms. In: Kyrö, P. \& Carrier, C. (eds.), The Dynamics of Learning Entrepreneurship in a Cross-Cultural University Context. Hämeenlinna: University of Tampere.

Klein, P. G., \& Bullock, J. B. (2006). Can Entrepreneurship be Taught? Journal of Agricultural and Applied Economics, 38(2), 429-439. https:// doi.org/10.1017/S107407080002246X

Koh, H. C. (1996). Testing hypotheses of entrepreneurial characteristics: A study of Hong Kong MBA students. Journal of Managerial Psychology, 11(3), 12 - 25. https://doi.org/10.1108/02683949610113566

Kourilsky, M.L., \& Esfandiari, M. (1997). Entrepreneurship education and lower socioeconomic black youth: an empirical investigation. The Urban Review, 29(3), 205-15. https://doi.org/10.1023/A:1024629027806

Rasmussen, E., \& Sørheim, R., (2006). Action-based entrepreneurship education. Technovation, 26, 185-194.https://doi.org/10.1016/j. technovation.2005.06.012

Rauch,A., \& Frese, M. (2007). Let's put the person back into entrepreneurship research: A metaanalysis on the relashionship between bussiness owern's personality traits, bussiness creation, and sucess. European Journal of Work and Organizational Psychology, 16 (4), 353-385.https://doi. org/10.1080/13594320701595438

Rico, M. I. L., Cámara, C. P., \& Llamazares, M. C. E. (2015). Impacto de la educación en el emprendimiento. Making-of y análisis de tres grupos de discusión. Pedagogia Social, (25), 221-250. 
Robinson, P.B., Stimpson, D.V., Huefner, J.C. \& Hunt, H.K. (1991). An attitude approach to the prediction of entrepreneurship. Entrepreneurship Theory \& Practice, 15(4), 13-31.

Sánchez, J. C. (2010). Evaluación de la Personalidad Emprendedora: Validez Factorial del Cuestionario de Orientación Emprendedora (COE). Revista Latinoamericana de Psicología, 42(1), 41-52.

Sánchez, J.C. (2011). Entrepreneurship as a legitimate field of Knowledge. Psicothema, 23(3), 427-432.

Sánchez, J.C. (2013). The Impact of an Entrepreneurship Education Program on Entrepreneurial Competencies and Intention. Journal of Small Business Management. 51(3), 447-465. https://doi.org/10.1111/ jsbm. 12025

Sánchez, J. C. \& Hernández, B. (2016). Emprendimiento e innovación: estrategias, desarrollo y crecimiento sostenible. Santiago de Compostela, España: Andavira Editora

Sánchez, J. C., Lanero, A., \& Yurrebaso, A. (2005). Variables determinantes de la intención emprendedora en el contexto universitario. Revista de Psicología Social Aplicada, 15(1), 37-60.

Sesen, H. (2013). Personality or environment? A comprehensive study on the entrepreneurial intentions of university students. Education + Training, 55(7). 624 -640. https://doi.org/10.1108/ET-05-2012-0059

Scott, M. G., Rosa, P., \& Klandt, H., (1998). Educating entrepreneurs for wealth creation. Ashgate, Brookfield.

Shaver, K.G. (1987). Principles of Social Psychology (3a ed.). Winthrop: Cambridge, MA.

Shapero, A., \& Sokol, L. (1982). Social dimensions of entrepreneurship. Kent, C.A., Sexton, D.L. and Vesper, K.H. (Eds), Encyclopedia of Entrepreneurship, Prentice-Hall, Englewood Cliffs, NJ, pp. 72-90.

Sogunro, O.A. (2004). Efficacy of role-playing pedagogy in training leaders: some reflections. Journal of Management Development, 23(4), 355-71. https://doi.org/10.1108/02621710410529802 
Sullivan, R. (2000). Entrepreneurial learning and mentoring. International Journal of Entrepreneurial Behavior \& Research, 6(3), 160-75. https:// doi.org/10.1108/13552550010346587

The Entrepreneurial University: From Concept to Action. (2013). National Centre for Entrepreneurship in Education (NCEE), p. 59.

Thomas, P. (2001). Women entrepreneurs opt for equity ahead of growth. Wall Street Journal, B2.

Thorp, H., \& Goldstein, B. (2010). Engines of innovation: The entrepreneurial university in the twenty-first century. Chapel Hill, NC: University of North Carolina Press.

Toca, C. (2010). Consideraciones para la formación en emprendimiento: Explorando nuevos hábitos y posibilidades. Estudios Gerenciales, 26(117), 41-60. https://doi.org/10.1016/S0123-5923(10)70133-9

Uriarte, J., \& González, P. (2007). Métodos e instrumentos de evaluación psicológica de jóvenes emprendedores. España. Recuperado de: http:// www.psicologiacientifica.com/metodos-e-instrumentos-de-evaluacionpsicologica-de-jovenes-emprendedores/

Vera, P.H., Amaru, E., \& González, M. (2013). Concretando la tercera misión ( $3 \mathrm{~m})$ de la universidad pública regional impactos y percepciones de un proyecto de extensión caso universidad del magdalena. Clio America, $7(14), 135-152$.

Villarán de la Puente, F. (2014). Educación emprendedora en la educación básica regular. Lima: Perú, Sistema Nacional de Evaluación, Acreditación y Certificación de la Calidad Educativa (SINEACE).

Yaniz, C., \& Villardón, L. (2006). Planificar desde competencias para promover el aprendizaje. Bilbao: Universidad de Deusto Cuadernos del ICE 12.

Zhao, H., Seibert, S. E., \& Hills, G. E. (2005). The mediating role of selfefficacy in the development of entrepreneurial intentions. Journal of Applied Psychology, 90(6), 1265-1272. https://doi.org/10.1037/00219010.90.6.1265 